Dear Author,

Please, note that changes made to the HTML content will be added to the article before publication, but are not reflected in this PDF.

Note also that this file should not be used for submitting corrections. 


\section{Lissajous figure-based single-frame collimation technique}

\section{Q1 Francisco Jose Torcal-Milla*, Luis Miguel Sanchez-Brea, Jose Maria Herrera-Fernandez}

Universidad Complutense de Madrid, Applied Optics Complutense Group, Optics Department, Facultad de Ciencias Físicas,

Plaza de las Ciencias 1, 28040 Madrid, Spain

\section{A R T I C L E I N F O}

\section{Article history:}

Received 18 March 2015

Received in revised form 5 June 2015

Accepted 7 July 2015

Available online $\mathrm{xxx}$

Keywords:

Collimation

Diffraction

Metrology

\begin{abstract}
A B S T R A C T
An accurate collimation technique based on a double grating system is proposed. Transversal displacement of the grating is not required and then, automatic single-frame processing can be performed. Talbot self-images are projected onto a mask composed by several shifted diffraction gratings. A Lissajous figure is obtained with the signals acquired by a CMOS camera where the mask is simulated by software. The collimation degree is determined by measuring the ellipticity of the Lissajous figure. Visual or automatic procedures for simple and accurate collimation of a light source are proposed. Experimental results are obtained which show a resolution of $\delta \phi \approx 4.16 \mu \mathrm{rad}$ in the divergence of the beam when a lens with focal length $f=25 \mathrm{~mm}$ and diameter $D=20 \mathrm{~mm}$ is used for collimation.
\end{abstract}

(c) 2015 Published by Elsevier B.V.

\section{Introduction}

Accurate beam collimation is essential in experiments and applications. A high number of techniques have been proposed and developed for testing the collimation degree of beams. Most of them are based on interferometry [1-5] or on self-imaging techniques [6-9]. In addition, several techniques have been proposed in the last years [10-15]. In particular, several collimation methods based on self-imaging that do not need a lateral displacement of the grating have been proposed, being these devices simple an stable. In [11] one circular grating is used instead of a linear grating and in [12] the collimation degree of the beam is obtained by measuring the period of one self-image produced by an amplitude Ronchi grating and comparing it with that of the grating. Nevertheless, the experimental configuration needs to be performed very accurately, since the period of one self-image is compared to the period of the grating and misalignments or environmental variations may produce wrong and inaccurate results. For example, small angular misalignments of the grating may produce an improper determination of the collimation degree.

Another robust and accurate technique was proposed in [10]. In this technique the light beam passes through a diffraction grating of period $p$ and a mask located at a Talbot plane of the grating, situated at $z_{T}=2 p^{2} / \lambda,[3,8,16]$. The mask is composed by two diffraction gratings with the same period $p$ and displaced laterally a distance $p / 4$. Two photodetectors are placed just behind each grating

\footnotetext{
* Corresponding author.

E-mail address: fjtorcal@ucm.es (F.J. Torcal-Milla).
}

centered at a certain distance from the optical axis. Two signals are obtained in the photo-detectors by displacing the grating laterally. When the beam is collimated, both signals are shifted $90^{\circ}$ and the Lissajous figure results a circle. When the beam is not collimated the phase shift between the two signals is not exactly $90^{\circ}$ and the Lissajous figure becomes elliptical. The collimation degree can be determined automatically by measuring the phase shift between both signals. The main objection to this technique is that a continuous transversal displacement of the grating is required in order to obtain the complete Lissajous figure.

In this work we develop a new collimation technique based on that proposed in [10]. The beam collimation is achieved by using a diffraction grating and a mask formed by several amplitude Ronchi gratings with known lateral shift. The light beam passes through the grating and the mask, and several photodetectors (or a CMOS camera) are used to obtain a Lissajous figure from the data. The collimation degree of the beam is related to the ellipticity of the Lissajous figure. However, the shape of this Lissajous figure is not simple and we must fit the experimental data to a rectangle/ellipse curve [17]. We observe that for a point light source and considering geometrical approach, the Lissajous figure is almost a rectangle with curved corners. Nevertheless, diffractive effects make the Lissajous figure more rounded. Then, to determine its ellipticity, we fit the Lissajous figure to a rectangle/ellipse curve. The fitting parameters are used to measure the collimation degree. The parameters are obtained with a simple computation and automatic or visual collimation can be easily performed with this technique. When a point source is collimated using a lens with focal length $f=25 \mathrm{~mm}$ and diameter $D=20 \mathrm{~mm}$, a resolution of $\delta \phi \approx 4.16 \mu$ rad in the divergence of the beam is obtained. 


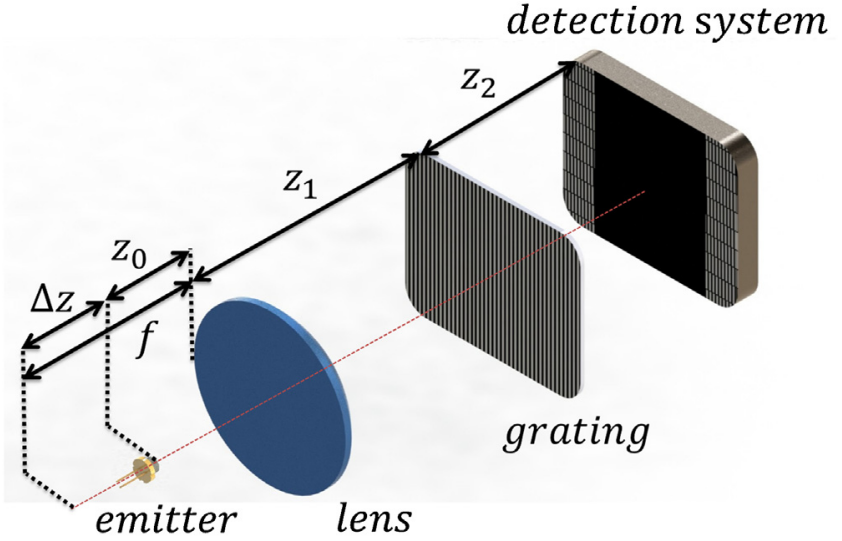

Fig. 1. Sketch of the proposed technique for beam collimation. $z_{0}$ is the distance from the emitter to the lens, whose focal length is $f, z_{1}$ is the distance from the lens to the diffraction grating $G, \Delta z=\left|z_{0}-f\right|$ is the distance from the emitter to the focal point of the lens, and $z_{2}$ is the distance from the grating to the mask $M$, where also photodetectors are placed behind.

Summarizing, the technique is explained and an analytical approach is performed in Section 2. In Section 3 we perform a numerical simulation of the technique and finally, in Section 4 we obtain experimental results which are in agreement with the theoretical frame.

\section{Analytical approach}

Let us consider the set-up shown in Fig. 1. It consists of a light source of wavelength $\lambda$ placed at a distance $z_{0}$ from the collimation lens with focal length $f$, a diffraction grating $G$ with period $p$, and a mask $M$ formed by several amplitude Ronchi gratings. The diffraction grating is placed at a distance $z_{1}$ from the lens and its transmittance is given by $t(x)=\sum_{n} a_{n} \exp (i q n x)$, where $n$ are integer numbers, $a_{n}$ are the Fourier coefficients of the grating, and $q=2 \pi / p$. For amplitude gratings, high-contrast self-images with the same period as the grating are observed at multiples of the Talbot distance, which is given by $z_{T}=2 p^{2} / \lambda$. At odd multiples of the semiTalbot distance, $(2 N+1) z_{T} / 2$, self-images of inverse contrast also appear. These odd self-images are also valid for applying the technique. When the emitter is exactly placed at the focal point of the lens, $\Delta z=\left|z_{0}-f\right|=0$, the beam after the lens is properly collimated and the period of the self-images is equal to that of the grating. On the other hand, when the emitter is not exactly at the focal plane, $\Delta z \neq 0$, the intensity distribution at a distance $z_{2}$ from the grating results in [10]

$I\left(x_{3}, z_{2}\right)=I_{0} \sum_{n, n^{\prime}} a_{n} a_{n^{\prime}} e^{i \frac{q}{1+\alpha z_{2}}\left(n-n^{\prime}\right) x_{3}} e^{-i \frac{q^{2}}{2 k}\left(n^{2}-n^{\prime 2}\right) \frac{z_{2}}{1+\alpha z_{2}}}$,

where $x_{3}$ is the coordinate parallel to the grating and perpendicular to the fringes at the observation plane, $I_{0}$ is the intensity of the incoming beam, $k=2 \pi / \lambda$, and $\alpha \approx-\Delta z / f^{2}$. As can be observed in the first exponential factor, the period of the self-images, $p_{\Delta z}$, depends on the collimation degree as

$p_{\Delta z}=\left(1+\alpha z_{2}\right) p \approx\left(1-\frac{\Delta z}{f^{2}} z_{2}\right) p$.

When $z_{2}=l z_{T}$ ( $l$ integer) the contrast of the self-images is maximum. Nevertheless, the technique does not need the observation plane to be exactly a Talbot plane to work, as we demonstrate in Section 3. The variation in the period shown in Eq. (2) produces a local phase shift of the fringes at locations out of axis. This phase shift can be easily detected with another diffraction grating with the same period placed at a distance $z_{2}$ from the first grating.
Instead of a simple grating, we propose the usage of the complex mask shown in Fig. 2a. It is formed by $M$ windows placed out of axis. Each window presents a diffraction gratings with the same period as the grating $G$ and a certain lateral displacement $\phi_{m}, m=1, \ldots, M$. The transmittance for each window is therefore $t_{m}^{\prime}\left(x_{3}\right)=\sum_{r} a_{r} \exp \left[i\left(q r x_{3}+\phi_{m}\right)\right]$, being $r$ integer numbers, $a_{r}$ the Fourier coefficients of the grating and $q=2 \pi / p$.

Then, the intensity distribution after each window $m$ is obtained by multiplying the intensity distribution times the transmittance, $I_{m}\left(x_{3}, z_{2}\right)=I\left(x_{3}, z_{2}\right) \cdot t_{m}^{\prime}\left(x_{3}\right)$, resulting in

$$
I_{m}\left(x_{3}, z_{2}\right)=I_{0} \sum_{n, n^{\prime}, r} a_{r} a_{n} a_{n^{\prime}} e^{i \frac{q}{1+\alpha z_{2}}\left(n-n^{\prime}\right) x_{3}} e^{-i \frac{q^{2}}{2 k}\left(n^{2}-n^{\prime 2}\right) \frac{z_{2}}{1+\alpha z_{2}}} e^{i\left(q r x_{3}+\phi_{m}\right)} .
$$

A photodetector is placed after each window in order to obtain the total intensity. Therefore the signal obtained with each photodetector results in

$S_{A, B}^{m}=\int_{x_{\min }}^{x_{\max }} I_{m}\left(x_{3}, z_{2}\right) d x_{3}$,

where $x_{\min }=x_{m}-\Delta x / 2$ and $x_{\max }=x_{m}+\Delta x / 2, x_{m}$ is the central position of the mth photodetector and $\Delta x$ is the photodetector size. Sub-index $A$ and $B$ determine pairs of related signals, as we will explain forward. Solving Eq. (4), the intensity collected by each photodetector is given by

$$
\begin{aligned}
S_{A, B}^{m}=I_{0} \Delta & x \sum_{n, n^{\prime}, r} a_{r} a_{n} a_{n^{\prime}} e^{-i \frac{q^{2}}{2 k}\left(n^{2}-n^{\prime 2}\right) \frac{z_{2}}{1+\alpha z_{2}}} e^{i \phi_{m}} \\
& \times \operatorname{sinc}\left[\frac{q \Delta x}{2}\left(\frac{n-n^{\prime}}{1+\alpha z_{2}}+r\right)\right],
\end{aligned}
$$

where $\sin c x=\sin x / x$.

A point in the Lissajous figure is obtained using two signals, $S_{A}^{m}$ and $S_{B}^{m}$, with a phase shift of $90^{\circ}$ between them, that is, displaced laterally $p / 4$. In Fig. 2a we can see an example of the proposed mask with $M=16$ windows ( 8 points of the Lissajous figure). Windows on the left side correspond to $S_{A}^{m}$ and windows on the right side correspond to $S_{B}^{m}$.

The best option is to include the highest number of windows as possible since then we will have more Lissajous points to determine the ellipticity. Nevertheless, a trade-off between the number of windows and the total size is required since, when the windows are very small, the signal/noise ratio $(S / N)$ obtained with each photodetector decreases. We have considered different number of windows and we have selected the option of $M=16$ windows as the best one for our set-up. Then, the mask is formed by diffraction gratings displaced $0,45,90,135,180,225,270$, and 315 electrical degrees for windows on the left, $S_{A}^{m}$, and 90, 135, 180, 225, 270, 315 , 0 , and 45 for windows on the right, $S_{B}^{m}$.

\section{Numerical simulations}

The Lissajous figure can be analytically obtained using Eq. (4) but we can use also a numerical approach. We have used a fast Fourier Transform based direct-integration method, [18], which uses the Rayleigh-Sommerfeld approach to determine the intensity distribution at the observation plane placed at a distance $z_{2}$ from the grating. Using this numerical approach, the optical intensity after the mask is shown, Fig. 2 b. This result is very similar to that obtained with the analytical approach, Eq. (5).

The points of the Lissajous figure are obtained integrating the intensity distribution for each window. The left windows are for the $x$-coordinate, $S_{A}^{m}$, and the right windows are for the $y$-coordinate, 
(a)

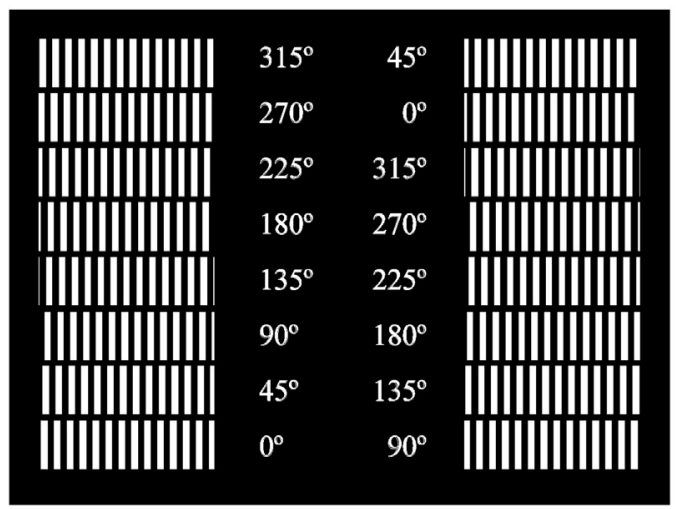

(b)

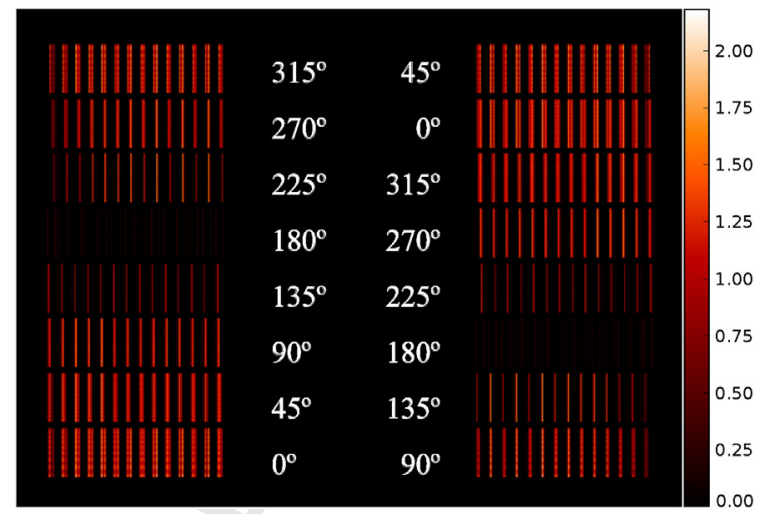

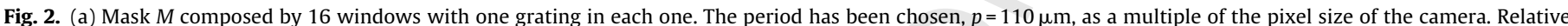

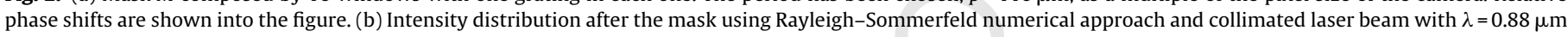
impinging a Ronchi grating.

(a)

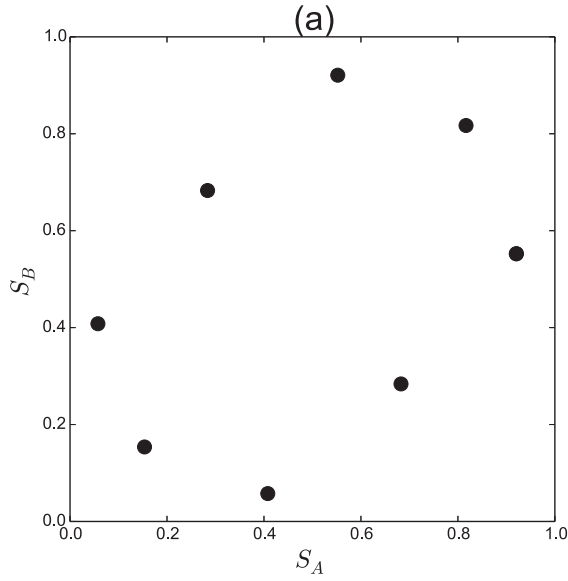

(d)

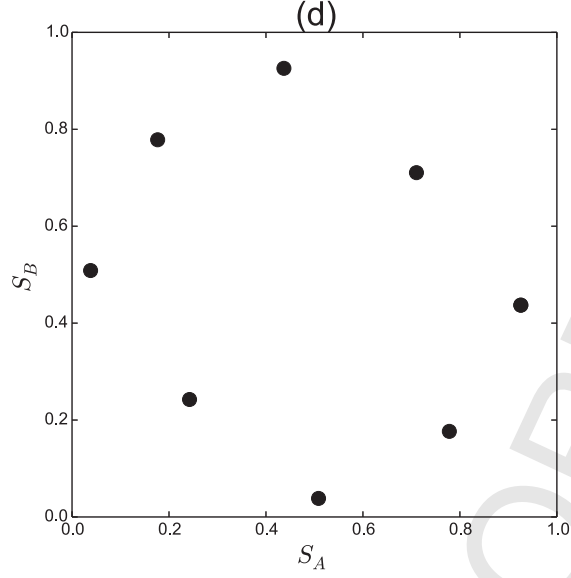

(b)

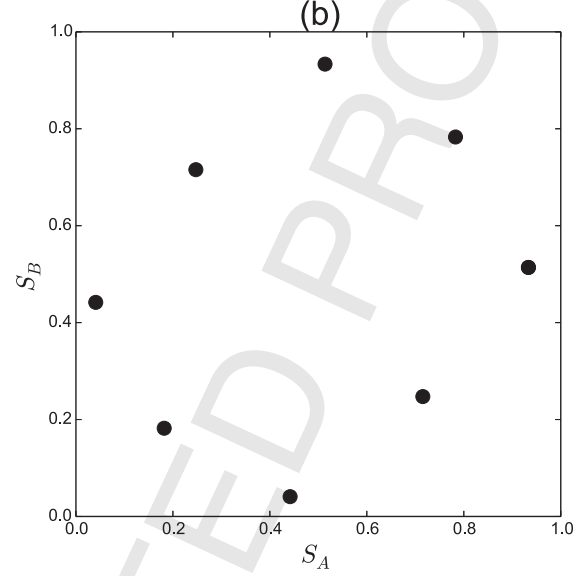

S

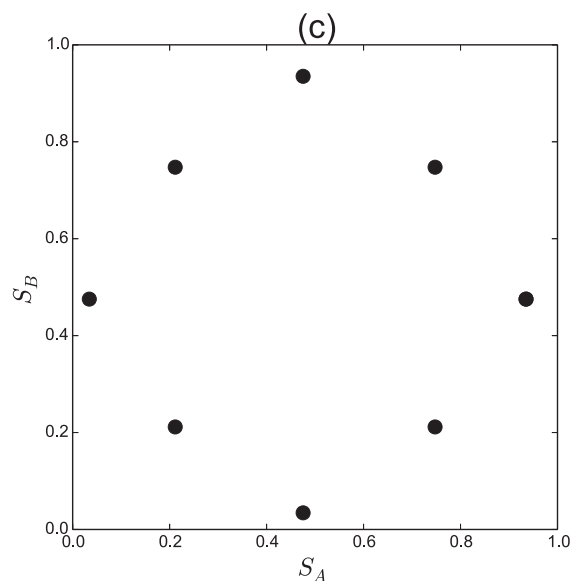

(e)

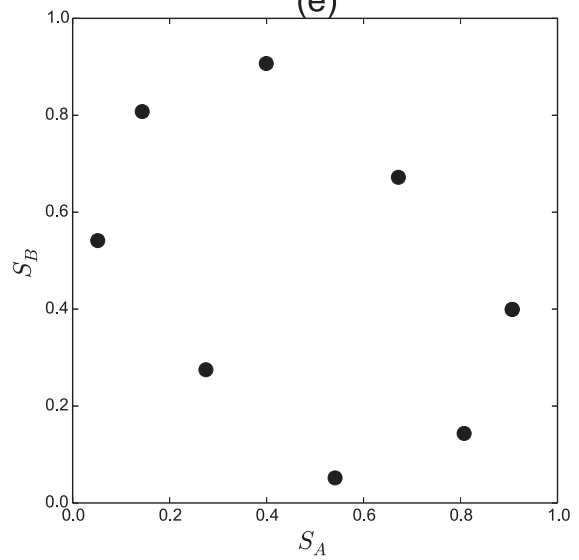

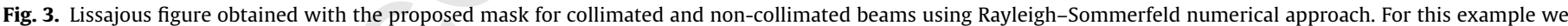

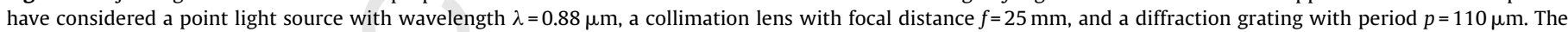

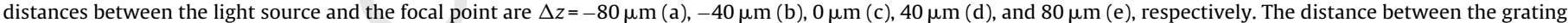
and the mask is $z=2 p^{2} / \lambda=27.5 \mathrm{~mm}$ We can see how the Lissajous figure becomes more elliptical as $|\Delta z|$ increases.

$S_{B}^{m}$. In Fig. 3 we can see several examples of the Lissajous figure for the cases of non-collimated and collimated beam. The numerical results are equivalent to those obtained using Eq. (5). When the incoming beam is perfectly collimated, the Lissajous figure is symmetrical, as that obtained in Fig. 3c. On the other hand, when the light source is not at the focus of the lens, the Lissajous figure becomes elliptical. As a consequence, the beam can be collimated simply looking at the Lissajous figure.

\subsection{Obtaining and fitting data to Lissajous figure}

Due to diffractive effects, the shape of the Lissajous figure is not a rectangle nor an ellipse but something in between when a point light source and a Ronchi grating are used. In order to properly determine the shape of the Lissajous figure, we have considered a squared/circular shape defined in [17] and we have modified it to a squared/elliptical shape 
(a)

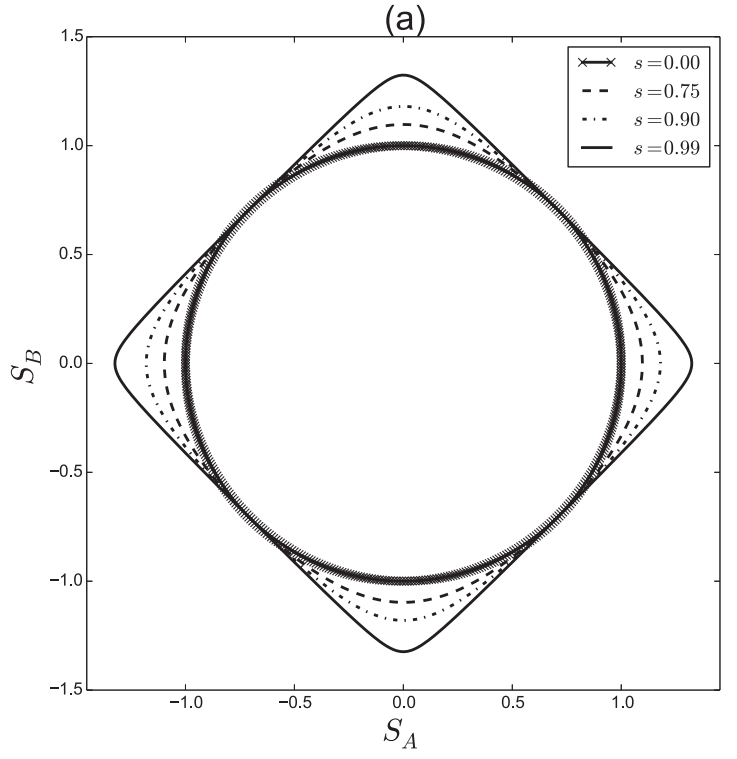

(b)

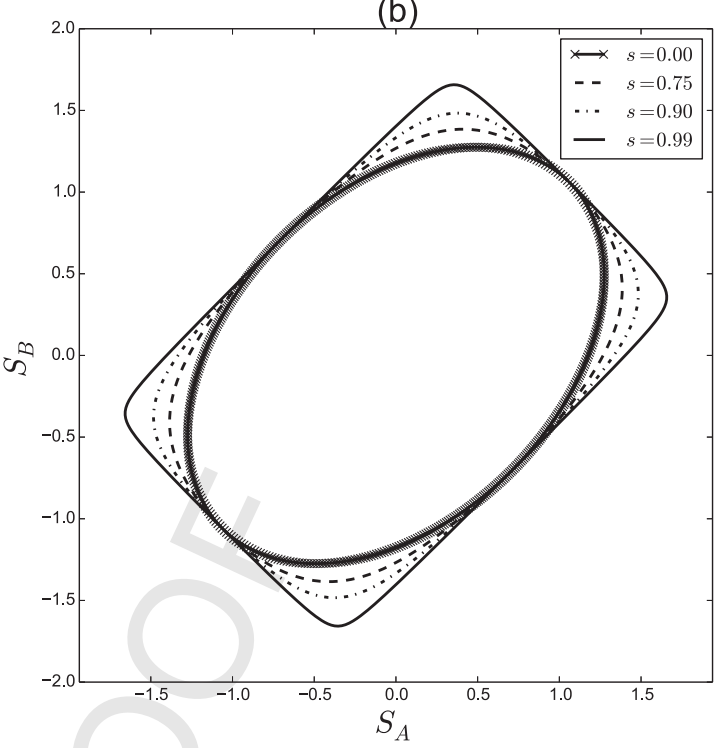

Fig. 4. Squared/circular shaped function for several values of $s$ parameter using Eq. (8). (a) The ratio $R_{1} / R_{2}=1$. (b) The ratio $R_{1} / R_{2}=1.5$.

$179 \quad \frac{x^{\prime 2}}{R_{1}^{2}}+\frac{y^{\prime 2}}{R_{2}^{2}}-s^{2} \frac{x^{\prime 2} y^{\prime 2}}{R_{1}^{2} R_{2}^{2}}=1$

where $R_{1}$ and $R_{2}$ are the lengths of the "principal axis" of the ellipse, and $s$ is a parameter that controls the squared/elliptical shape of the curve. When $s=1$, Eq. (6) represents an ellipse and when $s=0$ it represents a rectangle.

Besides, we need to include into the equation the facts that: (a) the principal axes of the ellipse are always placed at $\pi / 4$ with respect to the $x$ axis, and (b) the center of the ellipse is placed at the origin $(0,0)$. Then, we use the following change of variables

$x=\left(x^{\prime}-x_{m}\right) \cos \theta-\left(y^{\prime}-y_{m}\right) \sin \theta$,

$y=\left(x^{\prime}-x_{m}\right) \sin \theta+\left(y^{\prime}-y_{m}\right) \cos \theta$,

where $\theta=\pi / 4$ and $\left(x_{m}, y_{m}\right)$ is the mean value of the experimental data, and we obtain the following equation for the shape of the Lissajous figure

$$
\begin{aligned}
& \frac{s^{2}}{4 R_{1}^{2} R_{2}^{2}}\left(x^{2}-y^{2}\right)^{2}-\frac{1}{2}\left(\frac{1}{R_{1}^{2}}+\frac{1}{R_{2}^{2}}\right)\left(x^{2}+y^{2}\right) \\
& +\left(\frac{1}{R_{1}^{2}}-\frac{1}{R_{2}^{2}}\right) x y+1=0 .
\end{aligned}
$$

We show in Fig. 4 some examples of solution for this equation considering $R_{1}=R_{2}$ (Fig. $4 \mathrm{a}$ ) and $R_{1}=1.5 R_{2}$ (Fig. $4 \mathrm{~b}$ ).

In order to obtain the parameters of interest, $s, R_{1}$, and $R_{2}$, we must fit our experimental data $\left(S_{A}^{m}, S_{B}^{m}\right) \equiv\left(x_{m}, y_{m}\right)$ with $m=1, \ldots, M$ obtained with the experimental Lissajous figure to this square/ellipse function. For this, we will follow an strategy similar to [19]. Instead of the ellipse polynomial $a^{\prime} x^{2}+b^{\prime} x y+c^{\prime} y^{2}+d^{\prime} x+f y+g^{\prime}=0$, we will consider our simplified version

$a u+b v+c x y+1=0$,

where $u=\left(x^{2}-y^{2}\right)^{2}$ and $v=\left(x^{2}+y^{2}\right)$. Therefore, using matrix formalism, for each pair of values $\left(x_{m}, y_{m}\right)$ obtained from measurements we can represent the matrix coefficients of the Lissajous figure, A. On the other hand, $\mathbf{z}$ represents the vector of the coefficients and $\mathbf{b}$ represents the column vector with independent terms. Thus, Eq. (9) is rewritten as $\mathbf{A} \cdot \mathbf{z}=\mathbf{b}$,

$\left(\begin{array}{ccc}u_{1} & v_{1} & x_{1} y_{1} \\ u_{2} & v_{2} & x_{2} y_{2} \\ \vdots & \vdots & \vdots \\ u_{M} & v_{M} & x_{M} y_{M}\end{array}\right)\left(\begin{array}{l}a \\ b \\ c\end{array}\right)=\left(\begin{array}{l}-1 \\ -1 \\ -1 \\ -1\end{array}\right)$

where $u_{m}=\left(x_{m}^{2}-y_{m}^{2}\right)^{2}$ and $v_{m}=\left(x_{m}^{2}+y_{m}^{2}\right)$ with $m=1,2, \ldots, M$. The solution of the equations system is performed using an ordinary least squares fitting (Penrose-pseudoinverse),

$\mathbf{z}=\left(\mathbf{A}^{T} \cdot \mathbf{A}\right)^{-1} \mathbf{A}^{T} \mathbf{b}$.

With this equation we obtain the parameters $a, b$, and $c$ that best fit to the experimental data. In order to obtain the $s, R_{1}$, and $R_{2}$ parameters we have to compare Eq. (9) with Eq. (8). Then we obtain the following non-linear set of equations

$\frac{s^{2}}{4 R_{1}^{2} R_{2}^{2}}=a$,

$-\frac{1}{2}\left(\frac{1}{R_{1}^{2}}+\frac{1}{R_{2}^{2}}\right)=b$,

$\left(\frac{1}{R_{1}^{2}}-\frac{1}{R_{2}^{2}}\right)=c$,

which can be easily solved, resulting in

219

$R_{1}^{2}=\frac{-2}{2 b-c}$,

$R_{2}^{2}=\frac{-2}{2 b+c}$,

$s^{2}=\frac{16 a^{2}}{4 b^{2}-c^{2}}$. 
(a)

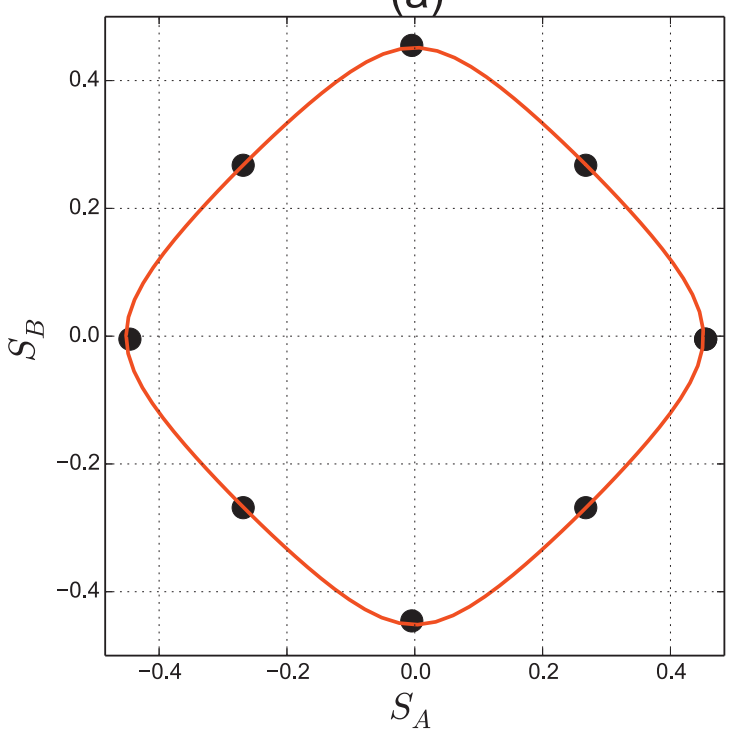

(c)

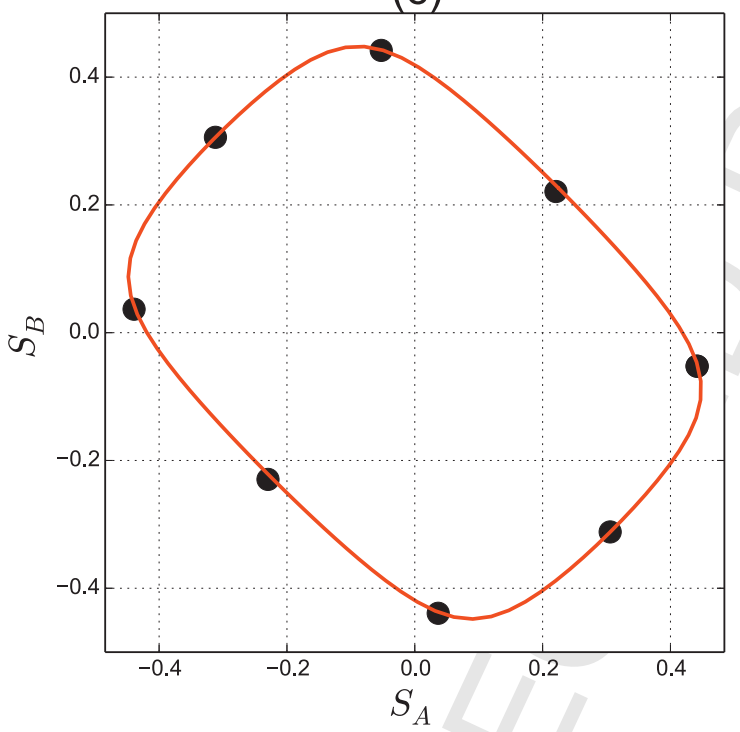

(b)

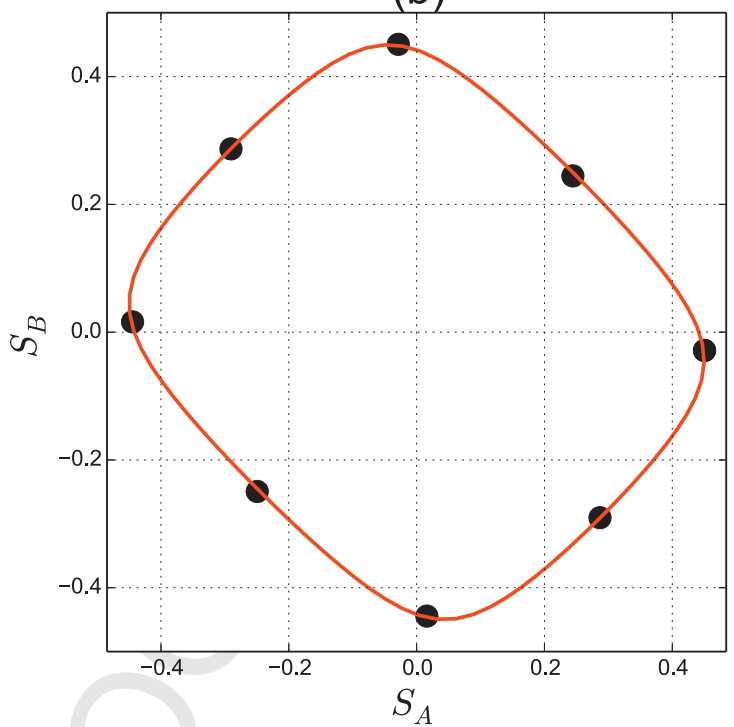

(d)

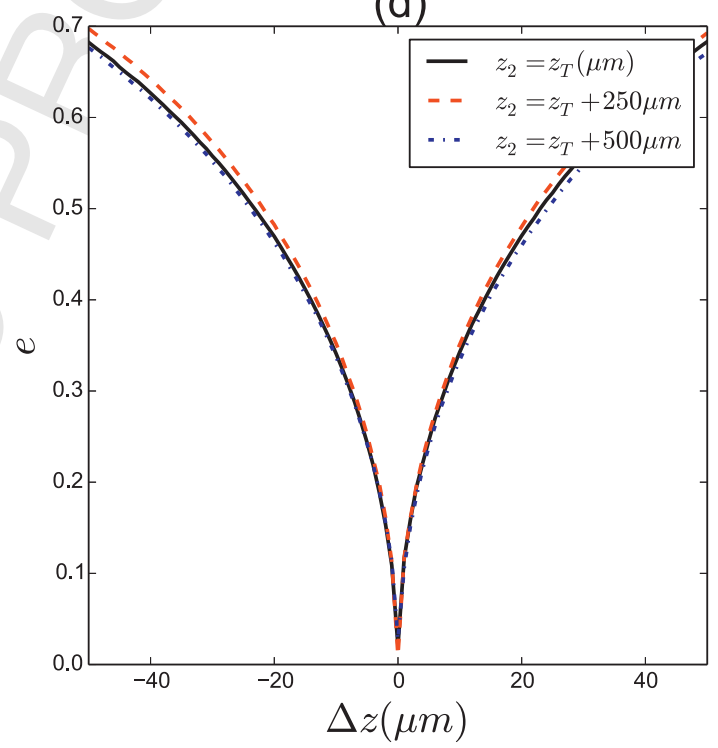

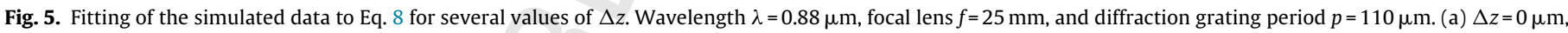

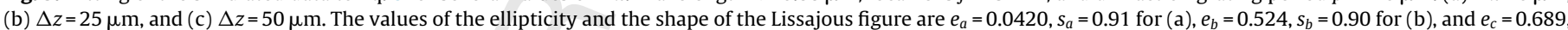

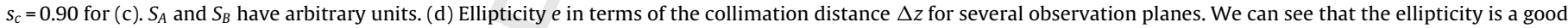
parameter for collimation since it is very sharp and it results almost zero for a collimated beam.

\subsection{Calculation of the ellipticity}

From Eq. (13), the collimation degree can be automatically determined by measuring the ellipticity of the Lissajous figure defined as

$e=\sqrt{1-\left(\frac{R_{\min }}{R_{\max }}\right)^{2}}$,

where $R_{\min }$ and $R_{\max }$ are the shorter and longer axes taken from $\left(R_{1}, R_{2}\right)$ respectively. When the ellipticity is zero, $e=0$, the beam is properly collimated. The first two parameters are used to determine the ellipticity, while $s$ is dependent on the shape of the Lissajous figure. As a consequence, the ellipticity is easily obtained resulting

$e^{2}=\frac{2 c}{2 b+c}$ or $\frac{-2 c}{2 b-c}$,

depending on whether $R_{1}$ or $R_{2}$ is the longer one.
As an example, in Fig. 5 we have performed three simulations using the Rayleigh-Sommerfeld approach. We have considered a point light source with wavelength $\lambda=880 \mathrm{~nm}$ and a diffraction grating with $p=110 \mu \mathrm{m}$. We have determined the Lissajous figure with the mask proposed in Fig. $2 \mathrm{a}$ and we have obtained the data, shown as dots. The Lissajous figure has been determined for three different distances $\Delta z$, (a) $\Delta z=0 \mu \mathrm{m}$ (b) $\Delta z=25 \mu \mathrm{m}$, and (c) $\Delta z=50 \mu \mathrm{m}$. Using the proposed approach we have determined the interpolated Lissajous figure considering Eq. (8). We can observe an excellent fitting for these values. We have also determined the ellipticity $e$ and the shape parameter $s$. For these three cases, the obtained parameters are $e_{a}=0.0420, s_{a}=0.91$ for $\Delta z=0 \mu \mathrm{m} ; e_{b}=0.524, s_{b}=0.90$ for $\Delta z=25 \mu \mathrm{m}$; and $e_{c}=0.689$, $s_{c}=0.90$ for $\Delta z=50 \mu \mathrm{m}$. The shape parameter $s$ is close to 1 and then the Lissajous figure is quite close to a squared shape. It means that we are observing at an integer number of $z_{T}$ from the first grating. 
(a)

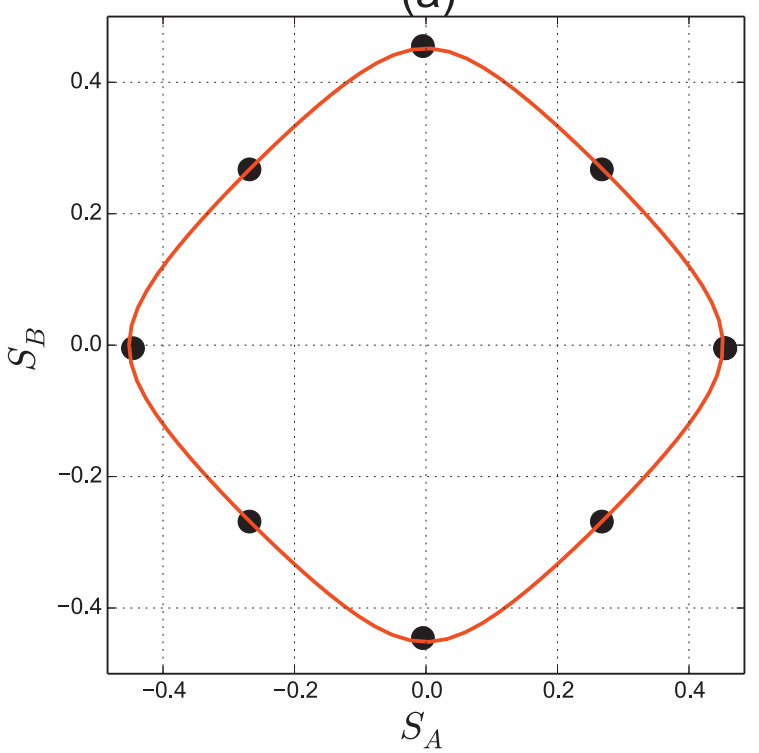

(c)

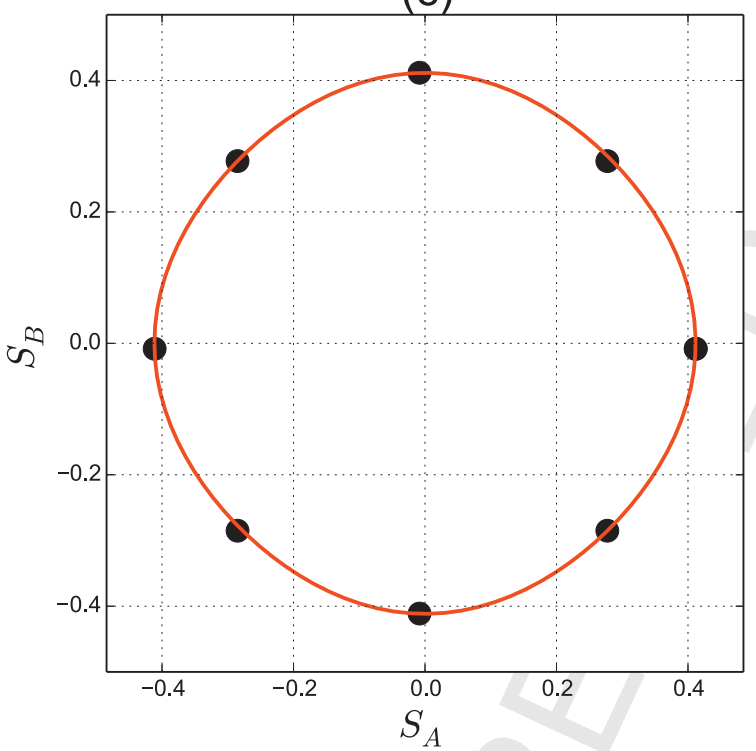

(b)

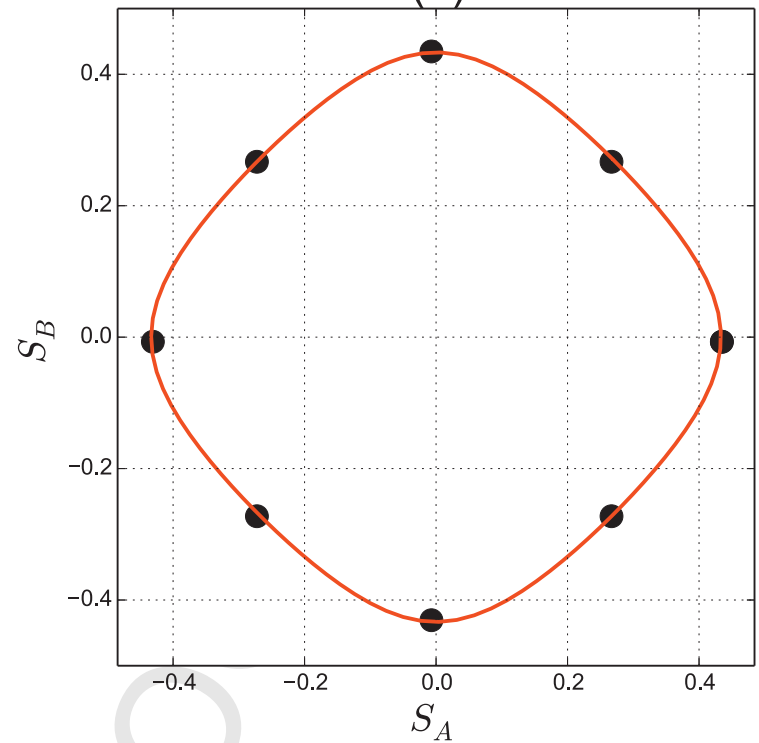

(d)

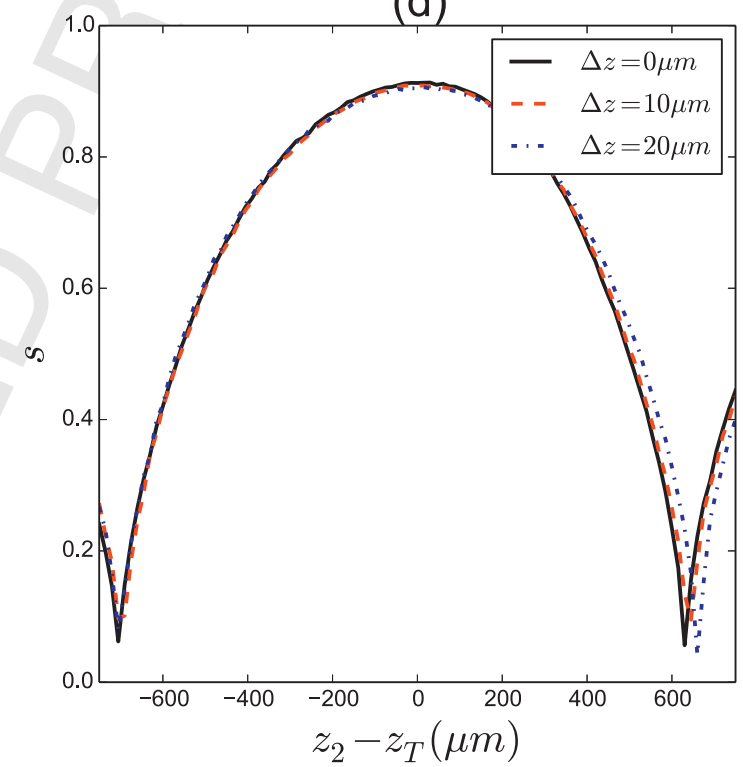

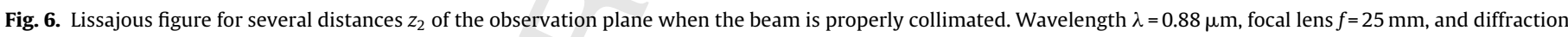

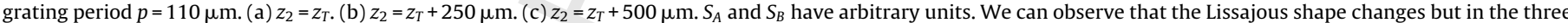

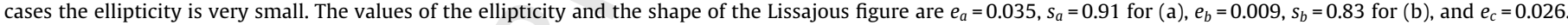

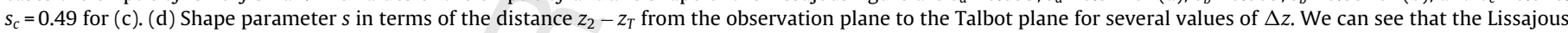
figure becomes circular as we separate from the Talbot plane.

In order to determine the validity of the proposed technique, we have numerically determined the ellipticity in terms of the distance $\Delta z$ between the light source and the focal point of the lens, Fig. $5 \mathrm{~d}$. We can observe that the ellipticity reaches a minimum for $\Delta z=0$, as it was expected. We can also see that this parameter is an excellent option for determining the collimation degree since it has a very sharp dependence, as was proposed in [10].

Finally, we analyze the tolerance in the location of the detection system with respect to the self-image location, $z_{2}$, by determining the Lissajous figure for several distances. In Fig. 6 we can see the simulated data for the case of $\Delta z=0 \mu \mathrm{m}$, and $z_{2}=z_{T}, z_{T}+250 \mu \mathrm{m}$, and $z_{T}+500 \mu \mathrm{m}$ respectively. We can see that the Lissajous figure becomes more circular as we separate from the Talbot/self-image plane. For these three cases, the shape parameters are $s_{a}=0.91$, $s_{b}=0.83, s_{c}=0.49$, respectively. The Lissajous figure changes but in the three cases the ellipticity is very small. In Fig. 6d we show this dependence for three different values of $\Delta z$. As can be observed, the three curves are very similar. It means that the observation plane is not a crucial parameter to measure the collimation degree of the beam. This fact could be useful to collimate sources with different wavelengths with the same device.

\section{Experimental results}

The experimental set-up is quite similar to that depicted in Fig. 1. In this case, we use a focused laser diode of wavelength $\lambda=650 \mathrm{~nm}$ that we collimate later with a bigger lens and a diffraction grating of period $p=110 \mu \mathrm{m}$. We choose this period due to the pixel size of the camera ueye UI-1480LE model by IDS, which is $2.2 \mu \mathrm{m}$. Thus, each period of the grating will correspond exactly to 
(a)

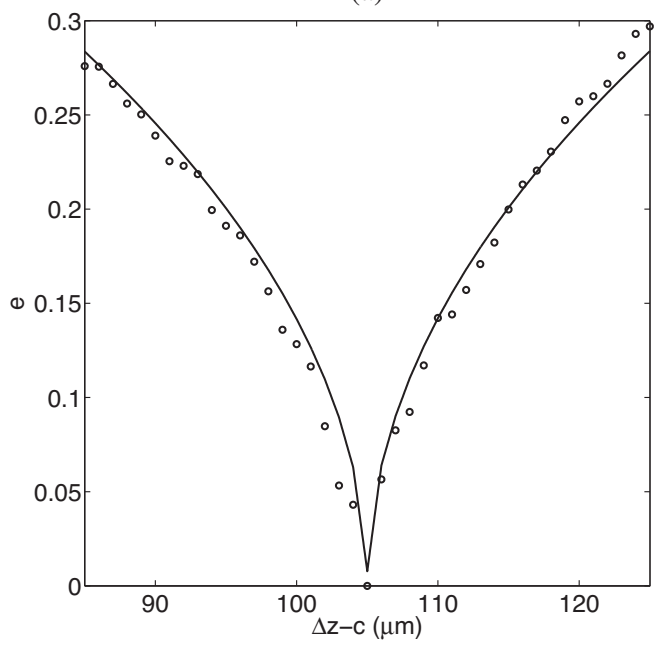

(b)

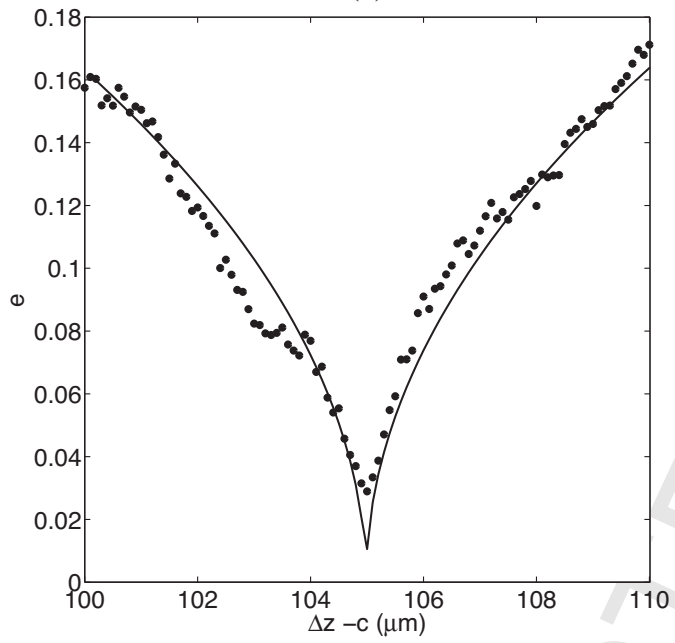

Fig. 7. Experimental (dots) and fitting (solid line) ellipticity $e$ in terms of the collimation distance $\Delta z$. Notice that $e$ is a dimensionless parameter. We have used as illumination source a focalized laser diode of wavelength $\lambda=650 \mathrm{~nm}$ and as collimating system a lens of diameter $D=20 \mathrm{~mm}$ and focal length $f=25 \mathrm{~mm}$. The grating and the mask have a period $p=110 \mu \mathrm{m}$ and $c$ denotes an unknown constant value due to the motorized stage initial position. (a) The measurements are taken by steps of $1 \mu \mathrm{m}$ displacement, (b) the measurements are taken by steps of $0.1 \mu \mathrm{m}$ displacement.
50 pixels of the camera and we avoid aliasing. Besides, we have enough pixels per period to obtain the fringes accurately. Collimation is performed along the axis perpendicular to the fringes of the grating. We use a collimation lens with focal length $f=25 \mathrm{~mm}$ and diameter $D=20 \mathrm{~mm}$. The moiregrams are acquired by the bidimensional CMOS camera and the mask is simulated by software, collecting the pixels according to Fig. 2a. We choose the first self-image that is placed at a distance $z_{2}=z_{T}=37.23 \mathrm{~mm}$ from the grating. The accuracy and sensitivity of the method increase with the self-image order number. Nevertheless, we have selected the first self-image for compactness and to compare directly the obtained results with those obtained in [10]. For the experiment, all elements are fixed except the illumination source (laser + focusing lens) which is displaced by using a motorized linear stage model M-511-DD by PI. The resolution given by the stage is $50 \mathrm{~nm}$ and the pitch error is around $\pm 30 \mu \mathrm{rad}$. The pitch error can be neglected for the considered longitudinal displacements. The experiment consists of displacing the source and computing the moiregram to obtain the ellipticity of the Lissajous figure. Once one has collected (a)
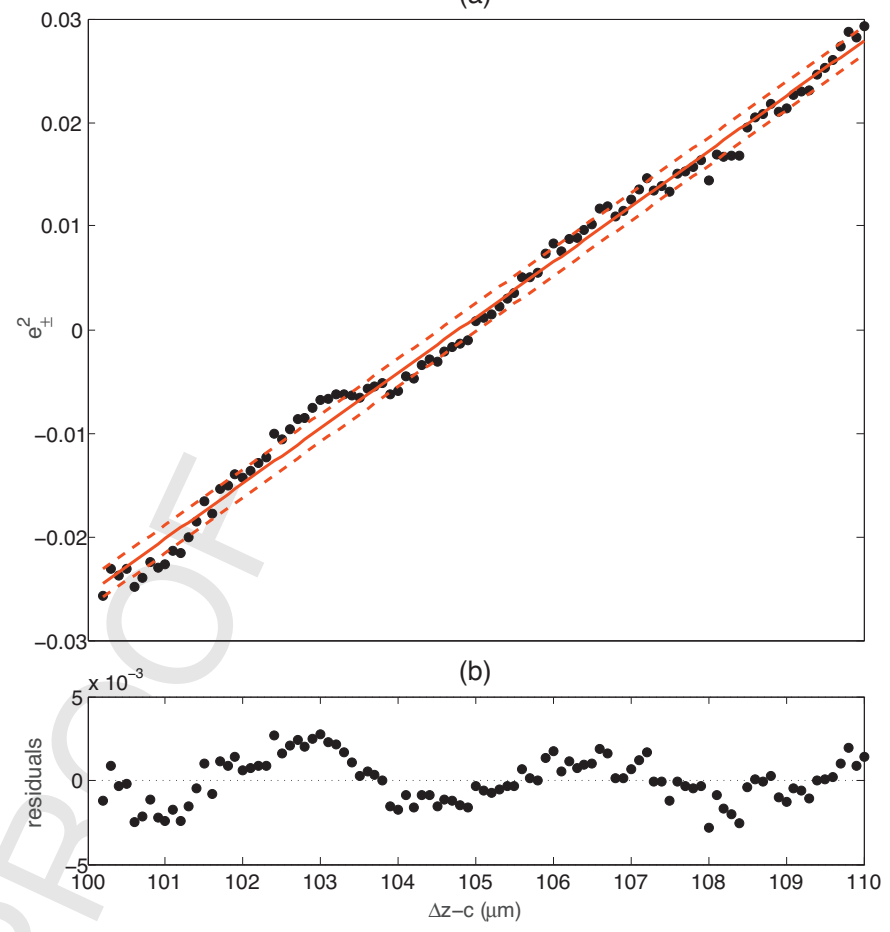

Fig. 8. (a) Experimental (dots) and linear fitting (red solid line) values for $e_{ \pm}^{2}$ in terms of the collimation distance $\Delta z$. Red thin lines represent the confidence interval. (b) Residuals of the linear fitting. The standard deviation of the residuals is $\operatorname{std}\left(e_{ \pm}^{2}\right)=\mathbf{Q} 4$ 0.0013 . (For interpretation of the references to color in this figure legend, the reader is referred to the web version of this article)

the ellipticity of the Lissajous figure for a range of distances, it is possible to obtain the relative position between source and lens that collimates the beam, $e=0$. Fig. 7 shows the experimental ellipticity of the Lissajous figure in terms of the distance point source-lens. In Fig. 7a the source is displaced by steps of $1 \mu \mathrm{m}$. As can be observed, the minimum of the ellipticity is clearly obtained. From the measurements, the lens can be placed with error lesser than one micron. In addition, Fig. $7 \mathrm{~b}$ shows the ellipticity by displacing the source by steps of $0.1 \mu \mathrm{m}$. Experimental results are affected by noise and this effect is better observed in Fig. $7 \mathrm{~b}$.

In order to analyze which is the uncertainty in the positioning of the light source with respect to the focal plane we have not used exactly Eq. (14) but a signed version of $e^{2}$, that is $e_{ \pm}^{2}=1-\left(R_{1} / R_{2}\right)^{2}$, where $R_{1}$ and $R_{2}$ are defined in Eq. (13). The value of $e_{ \pm}^{2}$ with this definition can be positive or negative depending on the ratio $R_{1} / R_{2}$. The parameter $e_{ \pm}^{2}$ depends linearly on $\Delta z$ as it is shown in Fig. 8a. We have performed a linear fitting to the experimental data shown in Fig. 8a, [20]. It results in $e_{ \pm}^{2}=a(\Delta z-\overline{\Delta z})+b$, with $a \pm \delta a=0.0053 \pm 0.0001, b \pm \delta b=0.0017 \pm 0.0003$ and $\overline{\Delta z}=105.1$. To measure the goodness of fitting we have determined the coefficient of determination ( $R$ squared), which results in $R^{2}=0.9925$, indicating an excellent fitting. The residuals of the fitting are shown in Fig. 8b, and the standard deviation of the residuals results $\operatorname{std}\left(e_{ \pm}^{2}\right)=0.0013$. The strategy to determine the uncertainty in the location of the emitter is by determining the uncertainty in the location of the value $e_{ \pm}^{2}=0$. Considering $\operatorname{std}\left(e_{ \pm}^{2}\right)$, the uncertainty in the location of the value $e_{ \pm}^{2}=0$ is $\delta z=0.26 \mu \mathrm{m}$ for a confidence interval of $95 \%$.

Using these data, we have also determined the minimum observable divergence, which results $\delta \phi=D \delta z / 2 f^{2} \approx 4.16 \mu \mathrm{rad}$. With this technique we improve in one order of magnitude the resolution in comparison with other very recent works. 
F.J. Torcal-Milla et al. / Sensors and Actuators A xxx (2015) $x x x-x x x$

\section{Conclusions}

In this work we show a new collimation technique which is based on a single-frame processing of the moiregram produced by two static diffracting objects, one diffraction grating and one more complex mask that we simulate by software in a CMOS camera. We obtain the corresponding Lissajous figure from each moiregram and calculate the ellipticity which gives the degree of collimation of the beam. With these results, a resolution of $\delta \phi \approx 4.16 \mu \mathrm{rad}$ in the divergence of the beam is obtained.

\section{Acknowledgements}

This work has been supported by the Ministry of Science and Innovation of Spain (project DPI2011-27851).

\section{References}

[1] M. Murty, The use of a single plane parallel plate as a lateral shearing interferometer with a visible gas laser source, Appl. Opt. 3 (4) (1964) 531-534.

[2] A. Ganesan, P. Venkateswarlu, Laser beam collimation using talbot interferometry, Appl. Opt. 32 (16) (1993) 2918-2920.

[3] D. Silva, A simple interferometric method of beam collimation, Appl. Opt. 10 (1971), 1980_1-1982.

[4] J. Choi, G.M. Perera, M. Aggarwal, R. Shukla, M. Mantravadi, Wedge-plate shearing interferometers for collimation testing: use of a moiré technique, Appl. Opt. 34 (19) (1995) 3628-3638.

[5] J. Darlin, M. Kothiyal, R. Sirohi, A phase-conjugate twyman-green interferometer with increased sensitivity for laser beam collimation, J. Mod. Opt. 45 (11) (1998) 2371-2378

[6] D. Joyeux, Y. Cohen-Sabban, High magnification self-imaging, Appl. Opt. 21 (4) (1982) 625-627.

[7] P. Senthilkumaran, Optical phase singularities in detection of laser beam collimation, Appl. Opt. 42 (31) (2003) 6314-6320.

[8] S. Yokozeki, K. Patorski, K. Ohnishi, Collimation method using fourier imaging and moiré techniques, Opt. Commun. 14 (4) (1975) 401-405.

[9] S. Haramaki, S. Yokozeki, A. Hayashi, H. Suzuki, Automatic collimation system by talbot interferometry, in: in: Optical Engineering for Sensing and Nanotechnology (ICOSN'01), International Society for Optics and Photonics, 2001, pp. 388-391.

[10] L.M. Sanchez-Brea, F.J. Torcal-Milla, F.J. Salgado-Remacha, T. Morlanes, I. Jimenez-Castillo, E. Bernabeu, Collimation method using a double grating system, Appl. Opt. 49 (17) (2010) 3363-3368.

[11] K. Patorski, K. Pokorski, M. Trusiak, Circular-linear grating talbot interferometry with moiré fresnel imaging for beam collimation, Opt. Lett. 39 (2) (2014) 291-294.

[12] L.M. Sanchez-Brea, F.J. Torcal-Milla, J.M. Herrera-Fernandez, T. Morlanes, E. Bernabeu, Self-imaging technique for beam collimation, Opt. Lett. 39 (19) (2014) 5764-5767.

[13] J. Dhanotia, S. Prakash, Automated collimation testing by incorporating the fourier transform method in talbot interferometry, Appl. Opt. 50 (10) (2011) $1446-1452$.

[14] R. Disawal, J. Dhanotia, S. Prakash, Improved measurement characteristics in collimation testing using lau interferometry and Fourier fringe analysis technique, Precis. Eng. 38 (4) (2014) 948-955.
[15] W.-Y. Chang, K.Y.Hsu, K.-H. Chen, J.-H. Chen, Collimation testing and calibration using a heterodyne moiré method, Opt. Lasers Eng. 62 (2014) 126-131.

[16] E. Keren, O. Kafri, Diffraction effects in moiré deflectometry, J. Opt. Soc. Am. A 2 (2) (1985) 111-120

[17] M.F. Guasti, M.D.L.C. Heredia, Diffraction pattern of a circle/square aperture, J. Mod. Opt. 40 (6) (1993) 1073-1080.

[18] F. Shen, A. Wang, Fast-Fourier-transform based numerical integration method for the rayleigh-sommerfeld diffraction formula, Appl. Opt. 45 (6) (2006) $1102-1110$.

[19] J. de Vicente, A. Sánchez-Perez, M. Berzal, P. Maresca, E. Gómez, Uncertainty in ellipse fitting using a flatbed scanner: development and experimental verification, Meas. Sci. Technol. 25 (1) (2014) 015005.

[20] JCGM, Evaluation of measurement data; guide to the expression of uncertainty in measurement (2008). URL http://www.bipm.org/utils/common/ documents/jcgm/JCGM_100_2008_E.pdf.

\section{Biographies}

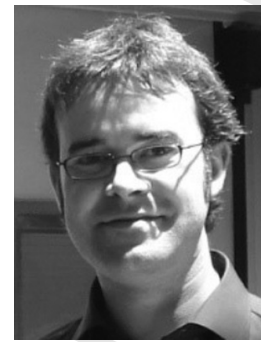

F.J.Torcal-Milla (Calatayud, Spain, 1980) received his MSc degree by the Universidad de Zaragoza (Zaragoza, Spain, 2004) and his Ph. D. degree by the Universidad Computense de Madrid, (Madrid, Spain, 2009). His research areas are related with metrology, optical encoders and computational vision. He has written more than 20 papers in these areas. He has been working in the Holography Group of the Universidad of Zaragoza and he is currently contracted by the Applied Optics Complutense Group of the Universidad Complutense de Madrid.

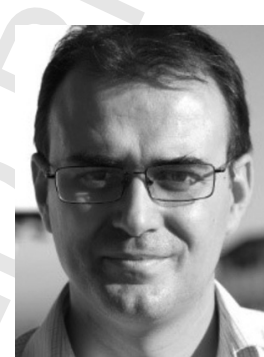

L.M. Sanchez-Brea (Madrid, Spain, 1972) received his MSc degree in 1996 and his PhD degree in Physics in 2001 from the Universidad Complutense de Madrid (Spain). His fields of research are Applied Optics Metrology and Image processing. He has written around 40 papers on these areas.He has been working at the Universidad Complutense de Madrid as Assistant Professor, in Fagor Automation S. Coop. as R+D technician, as "Ramon y Cajal" researcher at the Optics Department of the Universidad Complutense de Madrid. Currently he is Associate professor at this University.

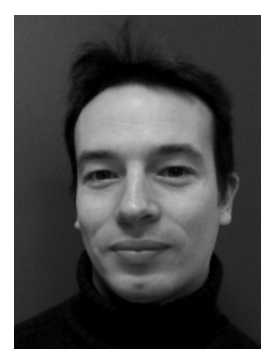

J.M. Herrera-Fernandez (Madrid, Spain, 1982) received his MSc degree from the Universidad Complutense de Madrid in Madrid, Spain, in 2008. Afterwards, he received his diploma in Applied Physics from the Universidad Complutense de Madrid in Madrid, Spain, in 2010. In 2009, he became a research fellow in the Applied Complutense Optics Group, AOCG, at the Universidad Complutense de Madrid. He worked in the design and analysis of diffractive optical elements for beam shaping and beam collimation testing. Nowadays, he is preparing his doctoral thesis. 